\section{MDS: Zytogenetik beeinflusst Prognose}

\section{Beim myelodysplastischen Syndrom (MDS) spielen zytogenetische Faktoren eine wichtige Rolle. Forscher befassten sich in einer aktuellen Studie mit deren Einfluss auf das Gesamtüberleben.}

$\mathrm{V}$ or Kurzem wurde beobachtet, dass ein monosomaler Karyotyp bei Patienten mit MDS mit einem schlechteren Gesamtüberleben assoziiert ist. Allerdings liegen hierzu widersprüchliche $\mathrm{Da}$ ten vor. Ein monosomaler Karyotyp ist definiert als 2 oder mehr autosomale Monosomien oder eine autosomale Monosomie und eine strukturelle Veränderung.

Nun wurde in einer populationsbasierten MDS-Kohorte untersucht, ob ein monosomaler Karyotyp unabhängig von der Zahl der zytogenetischen Veränderungen mit dem Gesamtüberleben assoziiert ist. Dazu wurden die Daten eines australischen Krebsregisters zu MDS-Fällen sowie Krankenakten der Kliniken analysiert. 1.404 MDS-Fälle, zu denen zytogenetische Ergebnisse vorlagen, wurden identifiziert. $65 \%$ der Pa- tienten hatten einen normalen, 9\% einen komplexen Karyotyp - definiert als 3 oder mehr zytogenetische Veränderungen. $7 \%$ wiesen einen sehr komplexen Karyotyp auf, d. h. 5 oder mehr Veränderungen.

Ein monosomaler Karyotyp war mit einem kürzeren medianen Gesamtüberleben in der gesamten Kohorte assoziiert (6 vs. 39 Monate; $\mathrm{p}<0,001)$. Das galt auch für Patienten mit einem gleichzeitig vorhandenen komplexen (monosomaler vs. nicht monosomaler Karyotyp: 6 vs. 17 Monate; $\mathrm{p}<0,001)$ oder sehr komplexen Karyotyp (monosomaler vs. nicht monosomaler Karyotyp: 6 vs. 9 Monate; $\mathrm{p}=0,02)$. Patienten mit der Kombination aus monosomalem und komplexem bzw. sehr komplexem Karyotyp hatten das kürzeste Gesamtüberle- ben. Auch nach der Adjustierung für die Anzahl an zytogenetischen Veränderungen blieb ein monosomaler Karyotyp mit dem Gesamtüberleben assoziiert, auch wenn die Effektstärke mit zunehmender zytogenetischer Komplexität abnahm: Die Hazard Ratio (HR) betrug für den monosomalen Karyotyp 4,81, für die Anzahl an zytogenetischen Veränderungen 1,22 und für die Interaktion zwischen einem monosomalen Karyotyp und zytogenetischen Veränderungen 0,83 .

Fazit: Diese Ergebnisse zeigen, dass der monosomale Karyotyp als unabhängiger Prädiktor für ein schlechteres Gesamtüberleben bei MDS-Patienten gelten kann, auch bei Patienten mit komplexem oder sehr komplexem Karyotyp. Allerdings nimmt der prognostische Effekt mit zunehmender zytogenetischer Komplexität ab.

Judith Neumaier

McQuilten ZK et al. Monosomal Karyotype Predicts Inferior Survival Independently of a Complex Karyotype in Patients With Myelodysplastic Syndromes. Cancer. 2015;121(17):2892-9.

\section{Hypomethylierer Decitabin: Hoffnung für MDS-Patienten mit hohem Risiko?}

\section{Die ersten Hinweise überraschten: Der Hypomethylierer Decitabin verbes- serte die Prognose älterer MDS-Patienten mit komplexem Karyotyp stärker als erwartet. In einer Phase-III-Studie wurde diese Beobachtung bestätigt.}

$E^{\text {in }}$ myelodysplastisches Syndrom (MDS) oder eine akute myeloische Leukämie (AML) mit komplexem Karyotyp sind v. a. bei älteren Patienten Hochrisiko-Konstellationen. Bei ihnen zeigten überraschend Clofarabin und die DNAHypomethylierer Azacitidin und Decitabin (DAC) hohe Aktivität. Warum nur diese Substanzen und nicht etwa auch Cytarabin und andere Zytostatika diesen Effekt zeigen, ist unklar. Mehr Klarheit auf klinischer Ebene lieferte jetzt die Analyse einer Kohorte von 206 älteren MDS-Patienten im Rahmen der EORTC/ GMDSSG-06011-Studie mit insgesamt 233 Patienten. Die untersuchten 206 Teilnehmer hatten randomisiert DAC oder Best Supportive Care (BSC) erhalten. Zy- togenetisch wurde bei ihnen u. a. der monosomale Karyotyp (MK) analysiert.

143 der Patienten wiesen zytogenetische Abweichungen (CA) mit MK-positivem oder -negativem Status auf, ein Teil erfüllte die Kriterien für ein hohes zytogenetisches Risiko. Endpunkte der Phase-III-Studie waren u. a. Gesamtansprechrate (ORR), progressionsfreies Überleben (PFS) und Gesamtüberleben (OS). Die ORR unter DAC betrug bei normaler Zytogenetik 36,1\%, bei MKnegativen Patienten 16,7\% und bei MKpositiven Patienten 43,6\% (MK1 44,4\%, MK2+ 43,3\%). Im Vergleich zu BSC verlängerte DAC das PFS sowohl bei zytogenetisch unauffälligen Patienten (Hazard Ratio [HR] 0,55, 99\%-Konfidenz- intervall [95\%-KI] 0,26-1,15; $\mathrm{p}=0,03$ ) sowie bei MK2+-Patienten (HR 0,50, $99 \%$-KI 0,23-1,06; $\mathrm{p}=0,016$ ), nicht jedoch bei den anderen Subgruppen (MK+, MK1, MK-). Das Gesamtüberleben verbesserte sich in keiner der Subgruppen.

Fazit: MDS-Hochrisikopatienten mit 2 oder mehr autosomalen Monosomien sprachen gut auf den Hypomethylierer Decitabin an. Ihr PFS war unter DAC im Vergleich zu Patienten unter BSC länger. Bei Patienten ohne oder mit nur einer autosomalen Monosomie war dies nicht der Fall. In weiteren Studien mit hypomethylierenden Substanzen sollte grundsätzlich der MK-Status vor Behandlungsbeginn erfasst und die hier gefundenen Zusammenhänge überprüft werden.

Barbara Kreutzkamp

Lübbert $M$ et al. Decitabine improves progression-free survival in older high-risk MDS patients with multiple autosomal monosomies: results of a subgroup analysis of the randomized phase III study 06011 of the EORTC Leukemia Cooperative Group and German MDS Study Group. Ann Hematol. 2016;95(2):191-9. 